\title{
Innovación y aprendizaje-servicio virtual: elementos para una reflexión basada en la experiencia
}

\author{
Juan García-Gutiérrez \\ Marta Ruiz-Corbella \\ Universidad Nacional de Educación a Distancia - UNED, España \\ Araceli del Pozo \\ Universidad Complutense de Madrid, España
}

\section{Resumen}

La innovación constituye una de las claves desde donde leer los desarrollos pedagógicos contemporáneos. En esta dirección, nuestro trabajo se aproxima a la noción de aprendizaje-servicio virtual como una modalidad innovadora que posibilita, justamente, la inclusión y el desarrollo de esta metodología en contextos educativos a distancia o virtuales. Concretamente, buscamos una definición basada en la experiencia. Por tanto, trataremos de reflexionar a partir del caso de un proyecto concreto de aprendizaje-servicio virtual sobre qué elementos y qué prácticas conexas ayudan a definir el recorrido de este tipo de proyectos. Consideramos que este tipo de aproximación y reflexión basada en la experiencia podría servir, además, para identificar aspectos que permitan y hagan posible su replicabilidad en otros contextos.

\section{Palabras clave}

Innovación, aprendizaje-servicio virtual, tecnologías digitales, ciudadanía global. 


\title{
Innovation and virtual service-learning: key elements for experience-based reflection
}

\begin{abstract}
Innovation is undoubtedly one of the key factors in assessing the best contemporary pedagogical outcomes. Our paper approaches the notion of virtual learning-service as an innovative modality that allows the inclusion and development of this methodology in distance or virtual educational environments. Specifically, we seek to provide a definition based on experience that also serves to identify elements and practices that allow others to start in this particular modality of service-learning. Therefore, we try to reflect on what elements and related practices help to define the development of this type of project. We believe that this reflexive approach would allow us to conceptualize the experience in a specific project of virtual learning-service and identify the key elements that would allow for replication in other contexts.
\end{abstract}

\section{Keywords}

Innovation, virtual service-learning, digital technologies, global citizenship. 


\section{El aprendizaje-servicio (en)clave de innovación en Europa}

La innovación educativa en la enseñanza superior es una de las líneas de investigación que, de forma interdisciplinar, más ha crecido y diversificado en las últimas fechas. La proliferación de congresos, jornadas así como los proyectos de investigación e innovación dedicados a esta intervención en las universidades reflejan este interés. Durante los últimos años, la investigación en innovación se ha convertido en una de las señas de identidad de las mejores instituciones de educación superior del mundo, lo que muestra un cambio en la mentalidad pedagógica institucional, producto de los ajustes y transformaciones que ha introducido en nuestro contexto europeo, especialmente a partir de la puesta en marcha del Espacio Europeo de Educación Superior.

En efecto, el proceso de Bolonia inaugura una etapa pedagógica nueva en la educación superior europea y también española. Cierto que no faltan críticas a sus planteamientos y que, para algunos, ha supuesto una cierta decadencia o universitaria (Llovet, 2011). Sin embargo, incluso aquellos más críticos, reconocerán que uno de los aspectos positivos de Bolonia pasa por haber situado la educación superior en el centro de la preocupación pedagógica y política. Si bien esta preocupación ha sido una constante en el tiempo, con Bolonia se renuevan las expectativas $y$, con ello, también la reflexión sobre su carácter educativo y

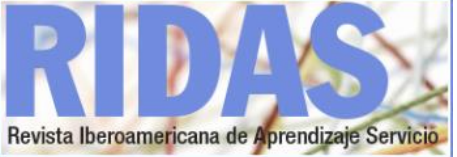

su necesaria implicación en la sociedad, su responsabilidad, lo que deriva en el conocimiento de la particular misión de aquellas instituciones donde se imparte (Esteban y Román, 2016).

Que la educación superior atienda a un complejo conjunto de competencias, reflejando así la globalidad del fenómeno educativo, lleva parejo la reflexión sobre el sentido y alcance de los elementos éticos y cívicos en los aprendizajes. Aunque para algunos este hecho significa cierta infantilización ${ }_{1}$ de las instituciones de educación superior (Furedi, 2018), desde nuestro punto de vista consideramos que la preocupación por esos elementos éticos y cívicos, que forman parte misma del fenómeno educativo, no quedan resueltos definitivamente o limitados a los niveles educativos inferiores al universitario. La educación sigue siendo tal, con independencia de la modalidad y del nivel educativo de que se trate.

Aunque, precisamente será la modalidad y el nivel educativo lo que permita ajustar y modular sus finalidades, contenidos, metodologías, etc.

Los políticos también han sabido ver en nuestras universidades como centros de innovación y creación de conocimiento, claves para una economía del conocimiento. Esta idea quedaba fijada en la Estrategia de Lisboa del 2000 y que posteriormente ha ido actualizándose de acuerdo al contexto cambiante en la política de esta región. Como es sabido, la estrategia de Lisboa

1 Tal y como reflejan también algunos titulares en prensa: "Universitarios poco adultos" (La Vanguardia, 2015).

García-Gutiérrez, J.; Ruiz-Corbella, M. y del Pozo, A. (2020). Innovación y aprendizaje-servicio virtual: elementos para una reflexión basada en la experiencia. RIDAS, Revista Iberoamericana de Aprendizaje Servicio, 9, 62-80. DOI10.1344/RIDAS2020.9.4 
apuntaba a hacer de la UE la economía basada en el conocimiento más competitiva y dinámica del mundo, capaz de crecer económicamente de manera sostenible con más y mejores empleos y con mayor cohesión social (Consejo Europeo, 2000). Ahora bien, tras la crisis económica de 2008, y con vistas a salir de ella de la mejor forma posible, la Unión Europea lanzaría en 2010 una visión renovada pero con una formulación muy similar. El objetivo se dirigió a que una Europa 2020 supondría una estrategia que ayude a salir fortalecidos de la crisis y convierta a la UE en una economía inteligente, sostenible e integradora que disfrute de altos niveles de empleo, de productividad y de cohesión social (Comisión Europea, 2010).

Esta visión de la economía del conocimiento ha puesto a los centros universitarios en el punto de mira de la estrategia político-económica de la UE y de los países miembros. Por ejemplo, la estrategia para la internacionalización de las Universidades españolas, del Ministerio de Educación, Cultura y Deporte (2015), abundaba en este planteamiento:

El conocimiento, es decir, la educación, la investigación y la innovación, se ha convertido en un importante motor de crecimiento y prosperidad, dada su capacidad para crear valor económico en el marco de un nuevo modelo de desarrollo socioeconómico basado en la inversión en capital humano y bienes intangibles [...] en este nuevo contexto la universidad está llamada a jugar un papel fundamental en cuanto que proveedora de capital humano cualificado, generadora de nuevo conocimiento y transmisora del existente, al ámbito productivo (p.14).

En cierta forma, con el Proceso de Bolonia se ha revitalizado y actualizado el debate entre los partidarios de una educación liberal y aquellos defensores de una educación vocacional; entre los defensores del papel que las humanidades pueden jugar en la construcción de la propia personalidad y la sociedad democrática y la necesidad de que los centros universitarios impulsen la innovación y la empleabilidad de los estudiantes. Ahora bien, actualmente no cabe considerar de forma antagónica estas posiciones sino que es preciso encontrar vías de conciliación y sinergia. Tan importante es formar a los estudiantes para saber conducirse en su vida personal, como para desarrollar con éxito una trayectoria profesional. Por tanto, no cabe decantarse a favor de una u otra posición sin poner en riesgo a la propia persona, en su identidad y proyecto vital. La búsqueda de una vida más plena conforme a las propias convicciones y un desarrollo profesional exitoso no deberían ser excluyentes.

Desde esta perspectiva, consideramos que el aprendizaje-servicio constituye no sólo una metodología innovadora sino una filosofía educativa que junto a otras modalidades como la investigación basada en la comunidad (Munck, McIlrath, Budd y Tandon, 2014) nos ayuda a comprender mejor la complejidad y riqueza del fenómeno educativo desde una posición 
integradora. Si tuviéramos que preguntamos el porqué del éxito del aprendizaje-servicio en las instituciones universitarias del contexto europeo, identificaríamos tres tipos de razones que, a la vez, nos ayudan a desmenuzar la complejidad actual por la que atraviesa la educación superior.

- En primer lugar, el aprendizajeservicio responde a la necesidad de encontrar y poner en marcha metodologías innovadoras que respondan a las demandas y postulados del Espacio Europeo de Educación Superior. La centralidad del estudiante, la promoción de aprendizajes activos y prácticos frente a clases magistrales, etc. constituyen buenas razones para indagar qué metodologías pueden adaptarse mejor a estos postulados.

- En segundo lugar, nos encontramos con la necesidad de profundizar y reforzar los aprendizajes éticos y cívicos en la educación superior (Bergan y Damian, 2010). En efecto, se constata desde hace décadas la solidez del movimiento por el compromiso cívico en la educación superior (Hartley, Saltmarsh y Clayton, 2010; McIlrath, 2015; Watson, Hollister, Stroud y Babcock, 2011), algo que también ha calado institucionalmente en el contexto europeo, como podemos comprobar por las recientes propuestas de la propia Comisión Europea. Así en la Comunicación de la Comisión titulada Sobre una agenda renovada de la UE para la educación superior de 2017 ( Comisión Europea, 2017) se dice que la Unión analiza:
"[...] la educación superior desempeña un papel único [...] para contrarrestar la creciente polarización de nuestra sociedad y la desconfianza hacia las instituciones democráticas, cada uno de nosotros, incluidos el personal y los estudiantes de educación superior, debemos comprometernos más activamente con las comunidades que nos rodean y promover la inclusión social y la movilidad (...) y que las instituciones de educación superior no sean torres de marfil, sino comunidades de aprendizaje con conciencia cívica conectadas a sus comunidades (párr.2).

- En tercer lugar, y fruto de las dos anteriores, encontramos la necesidad de las propias instituciones de educación superior por comunicarse y relacionarse con su entorno, desde un enfoque de responsabilidad social (Martínez, 2008). Preocupación constante por desarrollar ese carácter formativo, cívico y ético, y no únicamente profesionalizante, lo que exige "[...] una formación de todas las capacidades humanas, la promoción cultural, el desarrollo económico, técnico- 
científico, la consolidación de una ciudadanía democrática, con la misión de alcanzar, en definitiva, una sociedad con rostro humano" (Naval y Ruiz-Corbella, 2012, p.112).

Desde este horizonte, el aprendizajeservicio conduce a una valoración compleja del hecho educativo universitario sin reduccionismos y con una voluntad integradora. Una respuesta excelente a las demandas académicas, sociales y políticas que hoy vive la universidad y que lo han conducido a una importante expansión a lo largo de la geografía europea pero también en países y entornos culturales muy diversos. Expansión ya muy consolidada en Estados Unidos, Canadá y Latinoamérica, que han influido en la rápida extensión de esta metodología en universidades europeas, africanas y asiáticas. Prueba de ello fue la celebración del primer Congreso Europeo de Aprendizaje-Servicio en la Educación Superior (Madrid, 2018) donde se puso de manifiesto la gran apertura y recepción de esta metodología en nuestra región europea (de hecho, esta expansión europea del aprendizaje-servicio ya había comenzado con la celebración en 2016 y en 2017 del Congreso de la IARSLCE en Bolonia y en Galway, respectivamente).

A la extensión geográfica y cultural se suma también la puesta en marcha de modalidades novedosas en el desarrollo de los proyectos de aprendizajeservicio, atendiendo una característica de la educación de nuestro tiempo, que no cabe entenderla sin las tecnologías de la información y la comunicación, que abren escenarios digitales, cada vez más interconectados, en los que debemos disponer de los conocimientos y competencias necesarias para encontrar maneras de conectar redes, intereses y habilidades tanto en contextos online como offline, de tal modo que todo individuo pueda actuar e interaccionar en ellos como contextos de oportunidad.

\section{Modalidades innovadoras para el desarrollo del aprendizaje-servicio}

Para facilitar un acercamiento más completo al aprendizaje-servicio virtual es preciso detenernos, brevemente, en observar algunos elementos facilitadores y prácticas conexas de la educación digital. Aquellos elementos que favorecen el surgimiento de esta modalidad virtual son las tecnologías de la información y la comunicación y los dispositivos portátiles e inteligentes, precisamente, los mismos que están vinculados al crecimiento de esta educación virtual. Mientras que entre las prácticas conexas al aprendizajeservicio virtual cabría incluir, por ejemplo, el aprendizaje-servicio internacional o los proyectos de aprendizaje-servicio global.

\subsection{Aprendizaje-servicio internacional y aprendizaje-servicio global}

Desde la óptica del aprendizajeservicio, los proyectos pueden adoptar diversas modalidades según el énfasis o las características que se acentúen en cada uno de ellos. Tanto en la modalidad internacional como global, el aprendizaje-servicio articula y presta atención a la dimensión internacional 
de los aprendizajes, ya sea en clave transnacional o desarrollando las nociones de ciudadanía mundial y responsabilidad global. Sin embargo, la opción por una u otra modalidad no supone merma alguna en los elementos o rasgos esenciales que identifican los proyectos aprendizaje-servicio. Estamos ante la misma metodología ofrecida y desarrollada en contextos y con intencionalidades educativas diferentes.

Concretamente, a partir de estas modalidades podemos afirmar dos cosas. La primera, que la existencia de modalidades no supone algo distinto del aprendizaje-servicio tradicional, pero sí suponen un algo más, ya que acentúan una determinada característica, bien en el aprendizaje o bien en el servicio. Paradójicamente, la segunda cosa que puede decirse es que estas modalidades también ayudan a cuestionar pedagógicamente algunas nociones básicas del aprendizajeservicio tradicional. Por ejemplo, en estos casos, la noción de cercanía en la relación con la comunidad.

Como sucede con el aprendizajeservicio tradicional, estas modalidades también podría confundirse con acciones similares en las que la prestación de un servicio o el aprendizaje cuentan con ese componente internacional. Así, si ponemos el acento en la idea de servicio internacional podemos encontrar múltiples acciones que podrían asemejarse al aprendizajeservicio internacional. Entre otras, podemos encontrar las siguientes: la cooperación internacional, la cooperación universitaria para el desarrollo; el voluntariado internacional o el más reciente turismo solidario y sostenible. Si nos detenemos en analizar este tipo de acciones solidarias, que subrayan la dimensión internacional de la ayuda podemos encontrar algunas características comunes con el aprendizaje-servicio internacional. Por ejemplo, la cooperación internacional constituye un canal ya institucionalizado por el que los Estados ayudan a otros países, integrándose en la política exterior de los países que comprende todo un conjunto de acciones orientadas a la lucha contra la pobreza, el progreso humano sostenible y el mantenimiento de la paz. La cooperación universitaria al desarrollo, por su parte, constituye "una actividad diferenciada de otras orientadas de modo general a la internacionalización de la Universidad o a la cooperación internacional entre Universidades" (CRUE, 2006, p.4) que puede definirse, según el Código de conducta de las universidades en materia de cooperación internacional, como:
"el conjunto de actividades llevadas a cabo por la comunidad universitaria y orientadas a la transformación social en los países más desfavorecidos, en pro de la paz, la equidad, el desarrollo humano y la sostenibilidad medioambiental en el mundo, transformación en la que el fortalecimiento institucional y académico tienen un importante papel" (p.4).

Y, además, en la que la cooperación "enriquece humana y académicamente a las personas que participan en ella" (CRUE, 2006,p.4). 
Otra acción similar a las anteriores es el voluntariado internacional que, atendiendo a la cooperación al desarrollo, se distingue por no estar en manos del Estado, sino de organizaciones de la sociedad civil y de las organizaciones no gubernamentales. Esto ha interesado especialmente al ámbito universitario, que ha desarrollado este tipo de propuestas. Se trata de un voluntariado que se realiza fuera del país, colaborando y ayudando a organizaciones en muchos casos vinculadas a los sectores donde se realizan los estudios (educación, medicina, ingeniería, etc.).

Un movimiento novedoso que trata de establecer una sinergia entra las acciones de cooperación y voluntariado con el turismo es el turismo solidario. Esta actuación es recogida, por ejemplo, por la Agencia de Naciones Unidas para los refugiados (ACNUR), quien lo define en su propia web2 como una modalidad que conjuga el placer y la diversión del viaje, asociándolo a proyectos de cooperación. Se pretende que los turistas no sean meros espectadores de la realidad del lugar sino que se involucren de alguna manera que resulte beneficiosa para la población local. Esas características pueden ser compartidas tanto por las acciones anteriores como por el propio aprendizaje-servicio internacional:

- Sostenibilidad económica y ambiental. Este tipo de acciones, realizadas principalmente en

\footnotetext{
2 "Turismo solidario y sostenible: ¿qué ventajas tiene?" Disponible en:

https://eacnur.org/blog/turismo-solidariosostenible-ventajas/
}

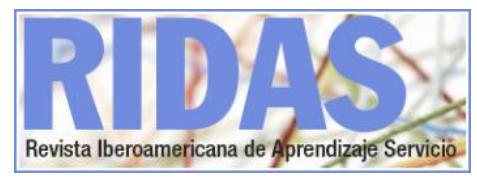

países en vías de desarrollo, tratan de tener un mínimo impacto ambiental, cuidando los entornos autóctonos.

- Promueve valores como el respeto, el cuidado y la justicia social. Quienes participan de estas acciones solidarias y sostenibles desarrollan conciencia mayor de las necesidades y dificultades que atraviesan las comunidades y los habitantes de otros países, ya que han convivido de cerca con sus necesidades. Se adquiere una mirada socialmente más sensible y responsable también al retornar al lugar de procedencia.

Tras lo dicho hasta ahora, podemos entender el aprendizaje-servicio internacional como una modalidad de aprendizaje-servicio donde la prestación del servicio se realiza en un país diferente al país donde se cursan los estudios, tratando de dar respuesta solidaria a las necesidades de una comunidad en un país distinto al que se cursan estudios. Lo relevante es cómo se establece la integración del servicio internacional dentro del plan de estudios. En esta modalidad es importante tener en cuenta el tiempo que durará la experiencia internacional, ya que es necesario contar con un tiempo razonable para que una experiencia de este tipo tenga sentido. $A$ la vez que exige una continuidad sostenible en el tiempo, mientras se alcanza el objetivo propuesto de cada proyecto.

Por tanto, una de las intenciones del 
aprendizaje-servicio internacional es abrir la mente al desarrollo de una responsabilidad global y hacer propios los problemas que tienen lugar más allá de las propias fronteras. Se busca es reforzar un sentido de ciudadanía global, responsabilidad y dialogo intercultural al conocer y permanecer inmerso durante un tiempo en una cultura distinta a la propia.

El límite más importante para este tipo de modalidad reside en la inseguridad de algunos países y en la financiación. Un ejemplo de este tipo de modalidad suele ser la salida de estudiantes de medicina, enfermería, educación, arquitectura o ingenierías a países en vías de desarrollo, donde pueden poner en práctica sus conocimientos desde el enfoque del aprendizaje-servicio o la investigación basada en la comunidad.

Por su parte, el aprendizaje-servicio global constituye, según Hastman y Kiely (como se citó en Morrinson, 2015):

"una experiencia de servicio impulsada por la comunidad que emplea prácticas estructuradas, críticas y reflexivas para comprender mejor la dignidad humana común; a uno mismo; la cultura; el posicionamiento; los problemas socioeconómicos, políticos y ambientales; las relaciones de poder y la responsabilidad social y todo en contextos globales [...] algo que ayuda a los estudiantes a aumentar la competencia intercultural, analizar suposiciones estructurales, comprender y reflexionar sobre la globalización y comprometer su imaginación cívica y moral ( $p$. 53).

Por tanto, el aprendizaje-servicio global apunta a la noción de ciudadanía global sin que esta intencionalidad suponga, necesariamente, un desplazamiento a otro país. El estudio de los fenómenos globales y la interdependencia socioambiental hacen de la Universidad un espacio pedagógico idóneo para desarrollar en los estudiantes un compromiso cívico que vaya más allá de visiones localistas y poder formar ciudadanos críticos globales (Larsen, 2014). Esto es, hacer posible a los estudiantes alargar los lazos del compromiso cívico a comunidades geográficamente distantes a la propia, desde la convicción de que aquello que ocurra allá apela también a la responsabilidad propia.

Para la Organización de las Naciones Unidas para la Educación, la Ciencia y la Cultura (UNESCO), tomar conciencia de esta común pertenencia es el elemento fundamental de una ciudadanía global. Esta idea de ciudadanía es la que trata de desarrollar esta perspectiva metodológica, sensibilizando sobre las problemáticas globales (por ejemplo la inmigración y los refugiados o el impacto del plástico en los océanos) y las posibilidades de actuación personal, cada vez más impulsados por los múltiples canales que nos facilitan las tecnologías.

2.2 Tecnologías digitales y aprendizajeservicio

La progresiva aplicación de las

García-Gutiérrez, J.; Ruiz-Corbella, M. y del Pozo, A. (2020). Innovación y aprendizaje-servicio virtual: elementos para una reflexión basada en la experiencia. RIDAS, Revista Iberoamericana de Aprendizaje Servicio, 9, 62-80. DOI10.1344/RIDAS2020.9.4 
tecnologías digitales a los procesos educativos es una constante que favorece tanto la innovación en las instituciones presenciales como la expansión sin precedentes de la educación a distancia y virtual (García Aretio y Ruiz Corbella, 2015). Por tanto, atender a los factores tecnológicos que posibilitan el desarrollo de una modalidad virtual del aprendizajeservicio implica preguntarnos, de alguna manera, por la forma en que las tecnologías digitales pueden intervenir o ser usadas en los proyectos de aprendizaje-servicio.

En efecto, las tecnologías digitales facilitan el desarrollo de los proyectos de aprendizaje-servicio, a la vez que propician el desarrollo de proyectos novedosos que abren paso a nuevas modalidades de aprendizaje-servicio. Esto es, las tecnologías digitales se pueden incluir en los proyectos de manera instrumental porque facilitan y optimizan el desarrollo de los proyectos, pero también pueden ser el objeto central del aprendizaje o del servicio.

Por ejemplo, si creamos una página web para que los estudiantes encuentren información sobre los proyectos y puedan registrarse online en ellos, estamos usando los recursos que nos ofrece la web 2.0 para impulsar proyectos de diversa índole. Ahora bien, si un proyecto se propone la reparación de dispositivos por parte de estudiantes de electrónica en la formación profesional para ofrecerlos a colectivos que los necesitan o cuando un grupo de estudiantes acompañan a personas mayores o personas inmigrantes para capacitarlos en el uso de las tecnologías digitales, como sucede con el programa Conectajoven, comprobamos que las tecnologías no sólo se están usando sino que tienen sentido, y se sitúan en el centro del proyecto al convertirse en canales de comunicación para interactuar entre dos colectivos con un objetivo común.

Como sucede en muchos ámbitos educativos puede resulta difícil diferenciar entre las tecnologías digitales como medios y como objetos de aprendizaje. Sin embargo, los proyectos basados en tecnologías digitales se distinguen por acentuar aquellos tipos de habilidades $y$ competencias que reclama la sociedad del conocimiento $y$, por tanto, prestan especial atención al desarrollo de la competencia digital y a las acciones que sólo pueden tener lugar en el ciberespacio. Los ejemplos anteriores nos muestran de manera sencilla de qué forma las tecnologías pueden enriquecer los proyectos de aprendizaje-servicio.

La inclusión de las tecnologías digitales en los proyectos de aprendizaje-servicio nos permite observar un rango amplio de interacciones, que llegan a identificar tres niveles o modos de interacción entres tecnologías digitales y el aprendizaje-servicio, recogidos en el siguiente cuadro.

3 "Proyecto Conecta joven". Disponible en: https://fundacionesplai.org/socioeducativa/cone cta-joven/

García-Gutiérrez, J.; Ruiz-Corbella, M. y del Pozo, A. (2020). Innovación y aprendizaje-servicio virtual: elementos para una reflexión basada en la experiencia. RIDAS, Revista Iberoamericana de Aprendizaje Servicio, 9, 62-80. DOI10.1344/RIDAS2020.9.4 
Cuadro 1. Niveles de integración tecnológica en los proyectos de aprendizaje-servicio

\begin{tabular}{|c|c|}
\hline $\begin{array}{c}\text { Nivel de } \\
\text { integración de } \\
\text { las tecnologías }\end{array}$ & Contenido \\
\hline $\begin{array}{l}\text { Integración } \\
\text { básica }\end{array}$ & $\begin{array}{l}\text { Las tecnologías digitales se } \\
\text { integran en los proyectos de } \\
\text { aprendizaje-servicio de } \\
\text { manera natural, ya que las } \\
\text { tecnologías facilitan la gestión } \\
\text { del proyecto. La introducción } \\
\text { no se establece con una } \\
\text { intencionalidad educativa sino } \\
\text { puramente instrumental o } \\
\text { facilitadora. }\end{array}$ \\
\hline $\begin{array}{l}\text { Inclusión } \\
\text { intencional }\end{array}$ & $\begin{array}{l}\text { Las tecnologías no sólo se } \\
\text { incluyen porque facilitan el } \\
\text { trabajo o mejoran la } \\
\text { visibilidad de los proyectos, } \\
\text { sino que existe una reflexión } \\
\text { pedagógica particular } \\
\text { tomándolas como objeto } \\
\text { educativo, bien desde la } \\
\text { perspectiva del aprendizaje o } \\
\text { del servicio. }\end{array}$ \\
\hline $\begin{array}{l}\text { Inmersión } \\
\text { tecnológica }\end{array}$ & $\begin{array}{l}\text { Las tecnologías digitales no } \\
\text { sólo están integradas en los } \\
\text { proyectos con una } \\
\text { intencionalidad educativa, } \\
\text { sino que el mismo proyecto se } \\
\text { desarrolla íntegramente en el } \\
\text { ciberespacio, apoyado en } \\
\text { recursos exclusivamente } \\
\text { digitales. Esto es, tanto el } \\
\text { aprendizaje como el servicio } \\
\text { es desarrollado de forma } \\
\text { online. }\end{array}$ \\
\hline
\end{tabular}

Fuente: elaboración propia

Podemos decir que la mayoría de los proyectos se sitúan en los dos primeros niveles. En cuanto facilitadoras, las tecnologías se incluyen en los proyectos de aprendizaje-servicio en tareas de comunicación, búsqueda de información o de creación de contenidos, por ejemplo. Algo que redunda en la mejora de los propios proyectos, la calidad de los aprendizajes y su difusión. Por tanto, las tecnologías se incluyen como parte de la gestión del proyecto pero también puede existir una intencionalidad educativa particular, como se comentaba en los ejemplos iniciales. En este sentido, para que dicha integración sea realmente pedagógica conviene realizar algunas preguntas: ¿de qué modo la incorporación de las tecnologías de la información y la comunicación potencia el aprendizaje curricular?, ¿de qué modo la incorporación de estas tecnologías promueve el protagonismo de los estudiantes para atender a los problemas de su comunidad?, ¿se podrían haber alcanzado los mismos resultados sin la incorporación de estas?, ¿cuál es el valor añadido que aportan las tecnologías de la información y la comunicación a los proyectos de aprendizaje-servicio? (Tapia Sasot, 2012).

En este sentido, cuando la inclusión de las tecnologías digitales cuenta además una intencionalidad educativa emergen diferentes relaciones. Por ejemplo, desde la organización pantallas amigas se ha desarrollado un programa denominado Cibermanagers 4 donde tras un proceso de capacitación los propios estudiantes se convierten en agentes de sensibilización para otros estudiantes y sus familias en materia

4 "Proyectocibermanagers". Disponible en: http://cibermanagers.com 
riesgos y uso seguro y responsable de internet. Como recoge Escofet (2017), los recursos tecnológicos pueden orientarse de diferentes formas: a la comunicación y la colaboración, a la búsqueda de información, hacia la creación de contenidos y para propiciar la reflexión. Para estos fines nos encontramos con recursos diferentes: videoconferencias, chats, foros virtuales, correo electrónico, Facebook, Twitter, wikis, blog, grupos de whatsapp, Drive, Dropbox, mapas conceptuales, infografías, etc.

Desde esta perspectiva, los proyectos de aprendizaje-servicio basados en tecnologías digitales nos permiten, además del desarrollo de las competencias digitales, ofrecer una apropiación ética y cívica de las mismas. En consecuencia, este tipo de proyectos nos permiten impulsar una visión humanista y humanizadora de las tecnologías digitales.

\section{Una noción de aprendizaje- servicio virtual basada en la experiencia}

Desde el horizonte de convergencia que hemos trazado anteriormente entre innovación pedagógica, tecnologías digitales y compromiso cívico se desarrollan los proyectos de aprendizaje-servicio puestos en marcha por el Grupo COETIC5 . A lo largo de los últimos años, han puesto en marcha diferentes proyectos de aprendizajeservicio virtual, entre los que destaca el

\section{Grupo de Innovación Docente para el} Desarrollo de la Competencia Ética y Cívica y las metodologías basadas en la comunidad en la educación superior de la UNED. proyecto Español en vivo6. Además de impulsar un programa específico para los Trabajos Fin de Grado y de Máster desde la investigación basada en la comunidad. Si en otras publicaciones hemos recogido el desarrollo de la experiencia del proyecto Español en vivo y sus resultados de aprendizaje, en este artículo pretendemos iniciar un proceso de reflexión y construcción, desde la experiencia, de la noción de aprendizaje-servicio virtual. Pero para ello es necesaria una breve descripción de nuestro proyecto.

Español en vivo promueve una serie de encuentros virtuales entre estudiantes universitarios españoles y africanos con el objetivo de hablar en castellano. El proyecto arranca con el viaje de una profesora española que se traslada a Benín, donde comienza a dar clases de español en la Escuela Normal Superior de Porto Novo (Benín). Tras compartir su experiencia sobre la situación educativa que se encontró; la falta de recursos pedagógicos de calidad, y la necesidad que manifiestan muchos de sus estudiantes de mejorar su español hablado, surge la idea de desarrollar un proyecto de aprendizaje-servicio que articule esa necesidad de los estudiantes africanos con el desarrollo de las competencias genéricas de los estudiantes españoles. Tras el primer curso, el proyecto se ha ampliado a otras Universidades como son la Universidad de Abomey-Calaví (Benín); Strathmore University (Nairobi, Kenia), y la Universidad de Dschang (Camerún).

6 Sitio web Grupo Coetic: https://www.uned.es/coetic/ 
Los estudiantes españoles están matriculados, en su mayoría, en la Universidad Nacional de Educación a Distancia (UNED) por lo que, desde el principio, el proyecto de aprendizajeservicio contaba con dos limitaciones, en relación con otros proyectos desarrollados en nuestro entorno. De un lado, los estudiantes no estaban todos juntos agrupados en un aula física, sino dispersos por todo el territorio español; de otro, la necesidad del colectivo al que se prestaba el servicio no estaba vinculada a una comunidad cercana. Ahora bien, estas limitaciones constituyen el punto de partida para el desarrollo de los proyectos de aprendizaje-servicio virtual, ya que la distancia (tanto en la agrupación de los estudiantes españoles, como en la relación con la comunidad) es salvada por la introducción de las tecnologías de la información y la comunicación.

Concretamente, el proyecto pretende incidir en el desarrollo de competencias éticas y cívicas de los estudiantes en un contexto educativo a distancia. Pero también, indirectamente, se trata de familiarizar a los estudiantes con la puesta en práctica de metodologías innovadoras, apoyados por las tecnologías digitales. Mostrar cómo aprovechar los entornos virtuales de enseñanza, característicos de la metodología a distancia para favorecer acciones solidarias. Lo que nos lleva a comprender la propuesta de los objetivos vinculados al aprendizaje:

- familiarizar a los estudiantes con el diálogo y la diversidad cultural;
- fomentar el compromiso con la ciudadanía global;

- tomar conciencia del compromiso ético en el desarrollo de la profesión;

- desarrollar la competencia digital y comunicativa.

Por un lado, los estudiantes de la UNED que cursan asignaturas que permiten participar en el proyecto, se encuentran distribuidos por diferentes regiones españolas. Estos cuentan con diferentes tipos de recursos para comunicarse online, lo que facilita una interacción digital que constituye uno de los elementos más relevantes del diseño de un entorno virtual de aprendizaje, que responde, según Mercado Borja, Guarnieri y Rodríguez (2019):

[...] a las diversas y cambiantes demandas de los participantes, a construir y democratizar el conocimiento, impulsar la innovación tecnológica, tributar al desarrollo integral del estudiante y a plantear alternativas de solución a problemáticas concretas desde espacios de discusión que permitan el flujo de conocimientos, ideas o información (p.73).

Se logra un aprendizaje mediado por la tecnología, vinculado a diferentes asignaturas de grado y postgrado, mediante la participación del estudiante y su interacción con los compañeros y los recursos disponibles en la plataforma aLF. Entre estos destacamos la Guía breve del proyecto que ofrece a

García-Gutiérrez, J.; Ruiz-Corbella, M. y del Pozo, A. (2020). Innovación y aprendizaje-servicio virtual: elementos para una reflexión basada en la experiencia. RIDAS, Revista Iberoamericana de Aprendizaje Servicio, 9, 62-80. DOI10.1344/RIDAS2020.9.4 
los participantes información básica para su participación en el proyecto (en qué consiste el aprendizaje-servicio y en qué consiste el propio proyecto, la descripción del servicio y de qué se habla en las entrevistas, plan de trabajo, etc.). Además de la Guía y otros recursos es importante mencionar el papel del foro, pues articula el trabajo colaborativo y las interacciones de los estudiantes a la hora de preparar las entrevistas, resolver dudas, plantear problemas, intercambiar experiencias sobre el proyecto y sus actuaciones. Desde el horizonte virtual que plantea el proyecto estos elementos son fundamentales para generar un clima de confianza y seguridad en las interacciones y en los aprendizajes y su relación con las asignaturas.

Por otra parte, el servicio también se desarrolla íntegramente en el ciberespacio. Esta vez, no es a través de una plataforma de e-learning específica sino mediante los diferentes programas que soportan comunicación online con video y voz (Whatsapp, Skype, etc.). El primer contacto se establece mediante correo electrónico con los coordinadores en las universidades africanas quienes indican al estudiante español el grupo de su universidad que le corresponde. La presencia de un coordinador en cada institución participante ayuda también a generar un ambiente de seguridad y confianza en el ciberespacio, además de facilitar el seguimiento, la reflexión y la evaluación del proyecto.

En suma, los proyectos de aprendizajeservicio virtual ponen en cuestión dos de los rasgos centrales de los proyectos de aprendizaje-servicio tradicionales, como son la cercanía y la presencialidad. Cercanía porque permite expandir la noción de comunidad (en línea con las propuestas del aprendizaje-servicio global o internacional) y presencialidad porque posibilita un contacto personal, pero no necesariamente síncrono y físico. Así, una vez visto que tanto el aprendizaje como el servicio se pueden conducirse íntegramente en el ciberespacio, podemos desarrollar brevemente aquellas características más relevantes que se inducen de la experiencia realizada con el proyecto:

- Creación de espacios de encuentro y confianza. En efecto, en primer lugar es fundamental apuntar a la creación de confianza entre todos los participantes. Los elementos que conforman el proyecto, así como aquellos otros que pudieran incluirse, deben estar orientados desde la perspectiva de los estudiantes, al encuentro y a la creación de espacios de seguridad y confianza online.

- La mediación tecnológica libera a los proyectos de aprendizajeservicio de los condicionamientos temporales y las limitaciones geográficas. Además, con la utilización de internet y dispositivos móviles se introduce la noción de aprendizaje ubicuo en el aprendizaje-servicio. De esta manera se facilita la interacción entre los participantes, dando lugar a formas de comunicación síncrona y asíncrona, diluyendo los espacios y acercando 
comunidades diferentes, incluso alejadas del lugar donde se desarrolla el aprendizaje. En este sentido, cabría la posibilidad de calificar también el aprendizajeservicio virtual como ubicuo, dado que tanto el servicio como el aprendizaje se pueden realizar desde cualquier lugar ya que en ambos casos se pueden desarrollar en el ciberespacio.

- Una tercera característica que observamos en el desarrollo del proyecto desde la modalidad virtual es el carácter solidario y humanizador que adquieren las tecnologías digitales, dado que propician la realización de una experiencia solidaria y humanizadora en el ciberespacio. De un lado, los fines del proyecto atienden al desarrollo de la competencia ética, el compromiso cívico y la ciudadanía global; de otro, la experiencia de la cooperación ya que esta no se limita a la ayuda material, sino que se abre a la solidaridad que se teje también de forma inmaterial.

- Por último, los proyectos de aprendizaje-servicio virtual permiten la inclusión ya que facilitan el acceso este tipo de proyectos innovadores a estudiantes y personas que por alguna razón (personales, familiares, profesionales) no pueden acceder a propuestas que se desarrollan presencialmente. A la vez que la accesibilidad que facilita la educación online permite también a los estudiantes con alguna discapacidad participar en esta modalidad.

Desde esta perspectiva, los proyectos de aprendizaje-servicio pueden adoptar una gran variedad de formatos atendiendo a la variable online, tal y como se recoge en el cuadro siguiente. Denominación que es calificada también por otros autores como aprendizajeservicio extremo (Waldner, McGorry y Widener, 2010).

Cuadro 2. Modalidades de aprendizaje-servicio online

\begin{tabular}{ll}
\hline \multicolumn{1}{c}{$\begin{array}{c}\text { Tipo de } \\
\text { aprendizaje- } \\
\text { servicio }\end{array}$} & \multicolumn{1}{c}{ Características } \\
\hline $\begin{array}{l}\text { Aprendizaje- } \\
\text { servicio } \\
\text { tradicional }\end{array}$ & $\begin{array}{l}\text { En este caso, tanto el } \\
\text { aprendizaje como el } \\
\text { servicio se desarrollan } \\
\text { presencialmente, en un } \\
\text { lugar determinado. }\end{array}$ \\
$\begin{array}{ll}\text { Aprendizaje } \\
\text { online - } \\
\text { servicio } \\
\text { presencial }\end{array}$ & $\begin{array}{l}\text { El aprendizaje se desarrolla } \\
\text { online, a través de un } \\
\text { curso en un espacio virtual } \\
\text { de aprendizaje, mientras } \\
\text { que el servicio se } \\
\text { desarrolla de forma } \\
\text { presencial. }\end{array}$ \\
& \\
$\begin{array}{l}\text { Aprendizaje } \\
\text { presencial - } \\
\text { servicio online }\end{array}$ & $\begin{array}{l}\text { El aprendizaje se desarrolla } \\
\text { de forma tradicional en un } \\
\text { aula mientras que el } \\
\text { servicio se desarrolla de } \\
\text { forma online. }\end{array}$ \\
\hline
\end{tabular}

García-Gutiérrez, J.; Ruiz-Corbella, M. y del Pozo, A. (2020). Innovación y aprendizaje-servicio virtual: elementos para una reflexión basada en la experiencia. RIDAS, Revista Iberoamericana de Aprendizaje Servicio, 9, 62-80. DOI10.1344/RIDAS2020.9.4 
Aprendizaje y servicio se desarrollan digitalmente. El aprendizaje se desarrolla de forma online, a través

Aprendizajeservicio virtual de un curso en un espacio virtual de aprendizaje, mientras que el servicio también se desarrolla de forma online, en el ciberespacio

Fuente: elaboración propia

Por otra parte, si donde ponemos el acento no es tanto en el ciberespacio sino en la noción de lugar (donde se producen los aprendizajes, y donde se realiza el servicio), si problematizamos este elemento de los proyectos de aprendizaje-servicio tradicionales podemos encontrar aun más posibilidades para el desarrollo de los proyectos. En efecto, al problematizar o extender la noción de lugar y cercanía, bien como aprendizaje-servico internacional, global o virtual nos encontramos con un enriquecimiento mayor de las experiencias realizadas por los estudiantes en los proyectos de aprendizaje-servicio (Snider, 2017). Idea que resumimos en la siguiente figura y que muestra las inmensas oportunidades que facilitan las tecnologías digitales a esta metodología.
Figura 1. Coordenadas que orientan la innovación en los proyectos de aprendizajeservicio

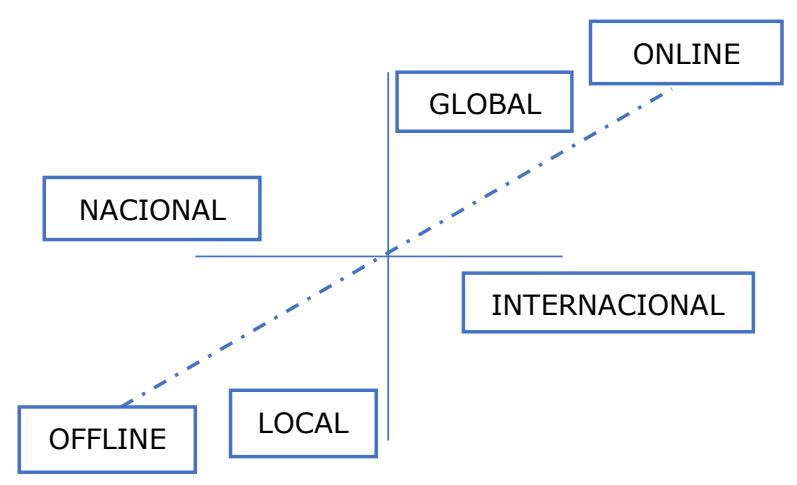

Fuente: elaboración propia

Tal como indicamos al inicio de este artículo, el objetivo de este trabajo era comenzar una reflexión sobre la conceptualización del aprendizajeservicio virtual y sobre el aprendizajeservicio basado en tecnologías digitales. En este punto, avanzamos ya una definición de aprendizaje-servicio virtual como una modalidad para el desarrollo de los proyectos de aprendizaje-servicio basada en internet y las tecnologías digitales, donde el aprendizaje y el servicio se realizan íntegramente en el ciberespacio.

En suma, como destacan diferentes instituciones internacionales, como la UNESCO o los últimos Informes del relator especial de Naciones Unidas sobre el derecho a la educación, es preciso afirmar el derecho a la educación y su sentido humanista frente a la comercialización y su función instrumental (o utilitarista) al servicio del desarrollo económico pero también frente a la creciente digitalización educativa. En efecto, en los citados 
informes, uno sobre la comercialización y otro sobre tecnologías digitales, el Relator recuerda que no debemos perder el carácter humanista de la educación ya sea en los escenarios digitales por presencia de las tecnologías digitales ni por la comercialización, lo que nos obliga a buscar, a hacer visible, esa dimensión humanizadora que deben mostrar las tecnologías digitales, ya que, en definitiva, son las personas las que humanizan todas sus acciones y actuaciones, mientras que "la tecnología es una herramienta, pero todas las herramientas están diseñadas con un propósito en la mente" (Breyfogle, 2018, 59).

\section{Referencias bibliográficas}

Agencia de Naciones Unidas para los refugiados. (Octubre de 2016). Turismo solidario y sostenible. ¿Qué ventajas tiene? [Mensaje en un blog].

Recuperado de https://eacnur.org/blog/turismosolidario-sostenible-ventajas/

Bergan, S. y Damian, R. (2010). Higher education for modern societies: competences and values. Strasbourg, France: Council of Europe Publishing.

Breyfogle, T. (2018). La tecnología como un medio para el progreso. En Aspen Institute España, Tech \& Society Un foro para pensar sobre el futuro de la sociedad tecnológica (pp.55-62). Madrid, España: Fundación Telefónica.

Comisión Europea. (2010). Comunicación de la Comisión. EUROPA 2020:Una estrategia para un crecimiento inteligente, sostenible e integrador. Bruselas: Comisión Europea. Recuperado de https://eurlex.europa.eu/legalcontent/ES/TXT/?uri=celex:52010DC20 20

Comisión Europea. (2017). Comunicación de la Comisión al Parlamento Europeo, al Consejo, al Comité Económico y Social Europeo y al Comité de las Regiones sobre una agenda renovada de la UE para la educación superior. Bruselas: Comisión Europea. Recuperado de https://eurlex.europa.eu/legalcontent/ES/TXT/HTML/?uri=CELEX:520 17DC0247\&from $=\mathrm{EN}$

Consejo Europeo. (2000). Sesión especial para acordar un nuevo objetivo estratégico de la Unión a fin de reforzar el empleo, la reforma económica y la cohesión social como parte de una economía basada en el conocimiento. Conclusiones de la Presidencia. Lisboa: Consejo Europeo de Lisboa.

Ministerio de Educación, Cultura y Deporte. (2015). Estrategia para la Internacionalización de las Universidades Españolas 2015-2020. Recuperado de: https://sede.educacion.gob.es/publiven $\mathrm{ta} / \mathrm{d} / 21475 / 19 / 1$

CRUE. (2006). Código de conducta de las Universidades en materia de cooperación al desarrollo. Recuperado de

http://ocud.es/es/files/doc512/codigoco nducta.pdf

Escofet, A. (2017). Aprenentatge servei i tecnologies digitals. Disponible en: https://www.fbofill.cat/publicacions/apr

García-Gutiérrez, J.; Ruiz-Corbella, M. y del Pozo, A. (2020). Innovación y aprendizaje-servicio virtual: elementos para una reflexión basada en la experiencia. RIDAS, Revista Iberoamericana de Aprendizaje Servicio, 9, 62-80. DOI10.1344/RIDAS2020.9.4 
enentatge-servei-i-tecnologies-digitals

Esteban, F. y Román, B. (2016). ¿Quo vadis, Universidad?. Barcelona, España: Universitad Oberta Cataluña (UOC).

Furedi, F. (2018). Qué le está pasando a la universidad. Un análisis sociológico de su infantilización. Madrid, España: Narcea.

García Aretio, L. y Ruiz Corbella, M. (2015). Educación a distancia, la perspectiva de la universidad en una sociedad en red: Una perspectiva desde las regiones de América Latina, El Caribe y América del Norte. Revista Española de Educación Comparada, 26, 11-14. Recuperado de http://revistas.uned.es/index.php/REEC /article/view/15819/13771

Hartley, M., Saltmarsh, J., y Clayton, P. (2010). Is The Civic Engagement Movement Changing Higher Education?. British Journal of Educational Studies, 58(4), 391-406. Doi:

$10.1080 / 00071005.2010 .527660$

Larsen, M. A. (2014). Critical Global Citizenship and International Service Learning: A case Studiy of the intensification Effect. Journal of Global Citizenship \& Equity Education, 4(1), 143. Recuperado de http://journals.sfu.ca/jgcee/index.php/j gcee/article/view/112/151

Llovet, J. (2011). Adiós a la universidad. El eclipse de las humanidades. Madrid, España: Galaxia Gutenberg.

Martínez, M. (Ed.). (2008). Aprendizaje Servicio y Responsabilidad Social de las
Universidades. Barcelona, España: Octaedro.

McIlrath, L. (2015). La Universidad cívica ¿un vacío legal y político?. Profesorado. Revista de currículum y formación del profesorado, 19(1), 2640. Recuperado de https://recyt.fecyt.es/index.php/profes orado/article/view/41020/23310

Mercado Borja, W., Guarnieri, G. y Rodríguez, G. (2019). Análisis y evaluación de procesos de interactividad en entornos virtuales de aprendizaje. Trilogía Ciencia, Tecnología y Sociedad, 11(20), 63-99.

doi: $10.22430 / 21457778.1213$

Morrison, E. (2015). How the I Shapes the Eye:The Imperative of Reflexivity in Global Service-Learning Qualitative Research. Michigan Journal of Community Service Learning, 22, 5266. Recuperado de https://quod.lib.umich.edu/cgi/p/pod/d od-idx/how-the-i-shapes-the-eye-theimperative-ofreflexivity.pdf?c $=$ mjcsl;idno $=3239521.0$ 022.105; format $=$ pdf

Naval, C. y Ruiz-Corbella, M. (2012). Aproximación a la responsabilidad social universitaria: la respuesta de la universidad a la sociedad. Bordón.

Revista de Pedagogía, 64(3), 103-115. Recuperado de https://recyt.fecyt.es/index.php/BORD ON/article/view/22053

Snider, M. (2017). Why 'where' Matters: Exploring the Role of Space in Servicelearning. Michigan Journal of Community Service Learning, 24, 3848. Recuperado de 
https://quod.lib.umich.edu/cgi/p/pod/d od-idx/why-where-matters-exploringthe-role-of-space-inservice.pdf?c $=$ mjcsloa; $i d n o=3239521.0$ 024.104; format $=$ pdf

Tapia Sasot, M. R. (2012). Las TIC en el desarrollo de los proyectos de aprendizaje-servicio. Recuperado de http://www.clayss.org/06_investigacion /jornadas/Libro_II-Jornada-

Investigadores-Aprendizaje-Servicio/CI09_TapiaSasot.pdf

Universitarios poco adultos. (15 de diciembre de 2015). La Vanguardia. Recuperado de https://www.lavanguardia.com/vida/20 151215/30811172390/universitariospoco-adultos.html

Waldner, L., McGorry, S. y Widener, M. (2010). Extreme E-Service Learning (XE-SL): E-Service Learning in the $100 \%$ Online Course. MERLOT Journal of Online Learning and Teaching, 6(4), 839-851. Recuperado de http://jolt.merlot.org/vol6no4/waldner 1210.pdf

Watson, D., Hollister, R., Stroud, S. y Babcock, E. (2011). The engaged University. International Perspectives on Civic Engagement. NY: Routledge. 